This is the accepted manuscript made available via CHORUS. The article has been published as:

\title{
Room-temperature relaxor ferroelectricity and photovoltaic effects in tin titanate directly deposited on a silicon substrate
}

Radhe Agarwal, Yogesh Sharma, Siliang Chang, Krishna C. Pitike, Changhee Sohn, Serge M. Nakhmanson, Christos G. Takoudis, Ho Nyung Lee, Rachel Tonelli, Jonathan Gardner, James F. Scott, Ram S. Katiyar, and Seungbum Hong

Phys. Rev. B 97, 054109 - Published 20 February 2018

DOI: 10.1103/PhysRevB.97.054109 


\section{Room-temperature relaxor ferroelectricity and photovoltaic effects in tin titanate directly deposited on Si substrate}

Radhe Agarwal, Yogesh Sharma ${ }^{2,3^{*}}$, Siliang Chang ${ }^{4}$, Krishna C. Pitike ${ }^{5}$, Changhee Sohn ${ }^{3}$, Serge M. Nakhmanson ${ }^{5}$, Christos G. Takoudis ${ }^{4,6}$, Ho Nyung Lee ${ }^{3}$, Rachel Tonelli, Jonathan Gardner ${ }^{7}$, James F. Scott ${ }^{8}$, Ram S. Katiyar ${ }^{1}$, and Seungbum Hong ${ }^{2,9 *}$

${ }^{1}$ Department of Physics and Institute for Functional Nanomaterials, University of Puerto Rico, San Juan, PR 00931, USA

${ }^{2}$ Material Science Division, Argonne National Laboratory, Lemont, IL 60439, USA

${ }^{3}$ Oak Ridge National Laboratory, Oak Ridge, Tennessee 37831, United States

${ }^{4}$ Department of Chemical Engineering, University of Illinois at Chicago, Chicago, Illinois 60607, USA

${ }^{5}$ Department of Materials Science and Engineering, Institute of Materials Science, University of Connecticut, Storrs, Connecticut 06269, USA

${ }^{6}$ Department of Bioengineering, University of Illinois at Chicago, Chicago, Illinois 60607, USA

${ }^{7}$ School of Chemistry, University of St. Andrews, St. Andrews, UK

${ }^{8}$ School of Physics and Astronomy, University of St. Andrews, St. Andrews, UK

${ }^{9}$ Department of Materials Science and Engineering, KAIST, Daejeon 34141, Republic of Korea

*Corresponding authors: sharmay@ornl.gov and seungbum@kaist.ac.kr 


\begin{abstract}
Tin titanate $\left(\mathrm{SnTiO}_{3}\right)$ has been notoriously impossible to prepare as a thin-film ferroelectric, probably because high-temperature annealing converts much of the $\mathrm{Sn}^{2+}$ to $\mathrm{Sn}^{4+}$. In the present paper, we show two things: first, perovskite phase $\mathrm{SnTiO}_{3}$ can be prepared by ALD directly onto p-type Si substrates; and second, these films exhibit ferroelectric switching at room temperature, with p-type $\mathrm{Si}$ acting as electrodes. X-ray diffraction (XRD) measurements reveal that the film is single-phase, preferred-orientation ferroelectric perovskite $\mathrm{SnTiO}_{3}$. Our films showed well-saturated, square and repeatable hysteresis loops of around $3 \mu \mathrm{C} / \mathrm{cm}^{2}$ remnant polarization at room temperature, as detected by out-of-plane polarization versus electric field (P-E) and field cycling measurements. Furthermore, photovoltaic and photoferroelectricity were found in $\mathrm{Pt} / \mathrm{SnTiO}_{3} / \mathrm{Si} / \mathrm{SnTiO}_{3} / \mathrm{Pt}$ heterostructures, of which properties can be tuned through band gap engineering by strain according to the first-principles calculations. This is a new lead-free room-temperature ferroelectric oxide of potential device application.
\end{abstract}




\section{INTRODUCTION}

$\mathrm{SnTiO}_{3}$ has been predicted to be a room-temperature ferroelectric with large polarization [1-3]. However, it has been difficult or impossible to process [4]. The Cambridge group found that only ilmenite formed [4], and in general, high-temperature processing and annealing may convert most of the $\mathrm{Sn}^{2+}$ to $\mathrm{Sn}^{4+}$. Therefore, we studied its production via lowtemperature ALD [5].

We emphasize that unlike ferromagnetism, ferroelectricity is not strictly a property of a material but of a system consisting of a dielectric and electrodes. In order to demonstrate switching it may be necessary, for example, to have blocking electrodes rather than ohmic, in order to minimize leakage currents and obtain useful hysteresis curves. In the present case, we demonstrate switching for $\mathrm{SnTiO}_{3}$ directly on conducting p-type $\mathrm{Si}$, which acts as both substrate and electrode. Si electrodes were first used in ferroelectric thin-film devices by the IBM San Jose group in the 1970s [6], and they are well understood. The main difference compared with metal electrodes is the longer screening length for depolarization fields [7].

There are known artifacts that can look like ferroelectric switching: L. Pintilie and M. Alexe [8] have shown that back to back diodes produce a polarization versus voltage P-V hysteresis curve that closely resembles true ferroelectricity. However, there are two ways to discriminate unambiguously between such artifacts and true ferroelectric switching hysteresis. First, artifacts consisting of charge injection have hysteresis curves that move clockwise with time [9], which is impossible for true ferroelectric hysteresis; and second, back-to-back diodes (for example, the $\mathrm{Si} / \mathrm{SnTiO}_{3}$ interfaces in our systems) will not exhibit a Curie temperature above which hysteresis disappears. In the present work, we demonstrate 
both of these requirements (counterclockwise hysteresis and a measured Curie temperature) to prove true ferroelectricity at room temperature.

There is no reason to be skeptical of the present results on $\mathrm{SnTiO}_{3}$ synthesis, since both the XRD and SEM [5] measurements closely match those reported previously from powder data $[10,11]$, which showed 100-nm diameter single-phase nanocrystals with $\mathrm{T}_{\mathrm{C}}=628 \mathrm{~K}$ (close to the frequency-dependent relaxor $\mathrm{T}_{\mathrm{C}}=600 \mathrm{~K}$ we measure). However, the earlier work was not commercially viable, since it requires a highly-toxic $\mathrm{TiCl}_{4}$ precursor. Moreover, $\mathrm{Sn}^{2+}$ in the powder prepared, due to disproportionation to $\mathrm{Sn}^{4+}$ and metallic $\mathrm{Sn}$, might not easily survive the sintering process requisite to make pellets or films for devices.

It has been reported that $\mathrm{SnTiO}_{3}$ has an optical band gap lying in the visible region of $\sim 2$ $\mathrm{eV}$ or even lower, which makes it a good candidate for photo-ferroelectric materials [12, 13]. Photo-ferroelectric materials are particularly interesting due to coupling between photosensitivity and ferroelectric properties, which provide a large possibility of optoelectronics and solar energy harvesting applications [14-18]. However, there are very few useful photo-ferroelectric materials because most ferroelectrics have wide optical band gaps $(>3 \mathrm{eV})$. Therefore, experimental values of the direct optical band gap in $\mathrm{SnTiO}_{3}$ thin films were also compared with theoretical values calculated from density functional theory (DFT). We have observed photo-ferroelectricity and a photovoltaic effect in our $\mathrm{Pt} / \mathrm{SnTiO}_{3} / \mathrm{p}$ $\mathrm{Si} / \mathrm{SnTiO}_{3} / \mathrm{Pt}$ capacitor structures. 


\section{METHODS}

\section{A. Experimental details}

$\mathrm{SnTiO}_{3}$ thin films of $40 \mathrm{~nm}$ thickness were deposited on p-Si substrates (p-type Si (100) substrates (resistivity: 1-10 $\Omega \cdot \mathrm{cm}, 20 \times 20 \mathrm{~mm}^{2}$ )) using atomic layer deposition (ALD) technique as detailed in reference [5]. Pt top electrodes were deposited on ALD-grown $\mathrm{SnTiO}_{3}$ thin films using RF-magnetron sputtering technique. X-ray diffraction (XRD) was performed with a PANalytical Empyrean diffractometer operating in reflection mode using monochromated $\mathrm{Cu} \mathrm{K} \alpha_{1}$ radiation. XRD data was collected from $2 \theta$ values of 10 to 120 degrees with step size of 0.017 degrees. Total data collection time was 90 minutes. Macroscopic ferroelectric properties were investigated with a ferroelectric loop tester (TF Analyzer 2000, aix-ACCT). To examine the intrinsic fatigue behavior, $10 \mu$ s wide electric pulses with a frequency of $10 \mathrm{kHz}$ were applied to the Pt-top electrode. An incandescent lamp was used as a source to measure light-induced changes in ferroelectric hysteresis loops. Photovoltaic measurements were performed using a Keithley-2401 electrometer under 1-sun AM 1.5 solar simulator with light source density $\sim 1 \mathrm{~kW} / \mathrm{m}^{2}$. Conduction mechanisms in $\mathrm{Pt} / \mathrm{SnTiO}_{3} / \mathrm{p}-\mathrm{Si} / \mathrm{SnTiO}_{3} / \mathrm{Pt}$ capacitors were studied through current-voltage (I-V) measurements using a Keithley 2401 source-meter unit. Temperature dependent measurements were done using a programmable Joule-Thompson thermal stage system (MMR model \# K-20). Dielectric measurements between frequencies $500 \mathrm{~Hz}$ and $300 \mathrm{kHz}$ were done using an impedance analyzer HP4924A. M-2000 ellipsometer (J. A. Woollam Co.) employed to record two ellipsometric parameters, $\psi$ and $\delta$, of $\mathrm{SnTiO}_{3}$ film on p-type $\mathrm{Si}$ substrate. Then a two-layer model composed of the substrate and film was used to determine 
the optical constants of the films. The optical constants of the substrate as well as film thickness were obtained separately and fixed during the fitting procedure.

\section{B. Theoretical methods}

A computational study was conducted to evaluate the bandgap of the polar perovskite $\mathrm{SnTiO}_{3}$ subjected to epitaxial strain following the approach outlined in reference [12]. DFT $[19,20]$ calculations were performed using the Vienna ab initio Simulation Package (VASP) $[21,22]$ within the local density approximation (LDA), parameterized by Perdew and Zunger [23]. The projector-augmented plane-wave method $[24,25]$ simulated the core and valence electrons. A plane-wave basis supporting the wave function cutoff at $900 \mathrm{eV}$ was used to converge the total energy of the system to within $1 \mathrm{meV} / \mathrm{f}$.u. Zone-edge-shifted $8 \times 8 \times 8$ Monkhorst-Pack (MP) [26] k-point mesh was used for the Brillouin zone (BZ) integration. Force components on individual ions were relaxed to less than $1 \mathrm{meV} / \AA$ and stress-tensor components on the simulation cell to less than $0.1 \mathrm{kbar}$ in all the structures studied. The monoclinic angle $(\beta)$ was not allowed to relax in the monoclinic $C m$ phase. The biaxial misfit

strain was defined as $\varepsilon=\frac{a}{a_{0}}-1$, where $a_{0}$ corresponds to the optimized lattice parameter of the cubic $\operatorname{Pm} \overline{3} m$ structure with all the normal stresses relaxed to values less than $0.1 \mathrm{kbar}$. It is well known that LDA exchange correlation functional underestimates the value of band gap. We have therefore computed more accurate values of the perovskite $\mathrm{SnTiO}_{3}$ band gaps using the Heyd-Scuseria-Ernzerhof (HSE) screened hybrid functional [27]. 


\section{RESULTS AND DISCUSSION}

X-ray diffraction (XRD) measurements were carried out to study the structural characteristics of our thin films. XRD patterns recorded at ambient conditions showed that the film is single-phase, preferred-orientation ferroelectric perovskite $\mathrm{SnTiO}_{3}$ (Figure S1). Based on the XRD data, we can predict that our films have grains with several orientations of (101), (011), (211), and (100) with c/a ratios of 1.144, 1.146, 1.036 and 1.061, respectively (see Supplemental Material) [28, 29].

Polarization-electric field (P-E) hysteresis loop measurements were done on $\mathrm{Pt} / \mathrm{SnTiO}_{3} / \mathrm{p}$ $\mathrm{Si} / \mathrm{SnTiO}_{3} / \mathrm{Pt}$ capacitors at different applied voltages, as shown in Fig. 1 (a). Inset of Fig. 1 (b) represents the schematic of $\mathrm{Pt} / \mathrm{SnTiO}_{3} / \mathrm{p}-\mathrm{Si} / \mathrm{SnTiO}_{3} / \mathrm{Pt}$ capacitor structure used for ferroelectric and photovoltaic measurements. P-E hysteresis loops in Fig. 1 (a) clearly indicate the presence of room temperature ferroelectricity with a well-defined remnant polarization and coercive field. The saturated $\left(\mathrm{P}_{\mathrm{s}}\right)$ and remnant polarization $\left(\mathrm{P}_{\mathrm{r}}\right)$ values were

found to be around $\sim 7.5 \mu \mathrm{C} / \mathrm{cm}^{2}$ and $3.3 \mu \mathrm{C} / \mathrm{cm}^{2}$, respectively, at room temperature and 40 $\mathrm{kHz}$ frequency.

From Fig. 1 (a), a clear hysteresis switching current characteristic with a nest of loops, one inside the other were observed with increasing applied voltages. The inset of Figure 1(a) displays the direction of the hysteresis curve as a function of time, which is counterclockwise and thus excludes the possibility of artifacts consisting of charge injection, which sometimes mimic ferroelectric-like behavior [9]. Some preliminary work on ferroelectric hysteresis was also done in an earlier report [5]. They switched via an AFM tip and obtained very reproducible fully saturated switching loops at a coercive field of $4 \mathrm{~V}$ across $40 \mathrm{~nm}$. This value of $E_{c}=100 \mathrm{MV} / \mathrm{m}$ is very reasonable for perovskite oxides and compares with about 
$20 \mathrm{MV} / \mathrm{m}$ in the present work. The present data are better than those of earlier work [5], because we have a parallel-plate capacitor structure and can therefore estimate electroded area, whereas no quantitative value of polarization could be obtained from the AFM-tip switched data of the earlier work. Perhaps most important in the present context, the hysteresis data of earlier work [5] are on an asymmetric $\mathrm{Si} / \mathrm{SnTiO}_{3} / \mathrm{AFM}$-tip geometry and not on a $\mathrm{Si} / \mathrm{SnTiO}_{3} / \mathrm{Si}$ symmetric sandwich. Therefore, the hysteresis data cannot arise from a back-to-back diode artifact, as discussed by Pintillie and Alexe [8]. We have also measured capacitance-voltage curves on single metal-oxide-semiconductor $\left(\mathrm{MOS}, \mathrm{Pt} / \mathrm{SnTiO}_{3} / \mathrm{Si}\right.$ ) and two back-to-back connected MOS $\left(\mathrm{Pt} / \mathrm{SnTiO}_{3} / \mathrm{Si} / \mathrm{SnTiO}_{3} / \mathrm{Pt}\right)$ based capacitor structures of $\mathrm{SnTiO}_{3}$ film (see Supplemental Material, Fig. S2) [28, 30-32], where the presence of two capacitance peaks attests to the fact that we are observing ferroelectric switching even in the MOS structure [30-32]. More interestingly, we observed the presence of two capacitance peaks (maxima) in more symmetric way for the case of two back to back connected MOS structure, revealing a true ferroelectric property of our $\mathrm{SnTiO}_{3}$ films.

Moreover, to exclude any possibility of charge injection and electret effect due to trapped charges at the $\mathrm{Si} / \mathrm{SnTiO}_{3}$ interface, we measured the frequency dependence of P-E hysteresis loops as shown in Figure 1 (c). Frequency dependence of P-E hysteresis loop illustrates clear polarization switching at higher frequencies (up to $80 \mathrm{kHz}$ ). Such frequency dependent behavior eliminates the possibility of a back-to-back diode effect, where the response at such high frequencies is not feasible for electrets since slow motion of ions is required. In addition, temperature dependent P-E hysteresis loop and polarization measurement using PUND technique were also performed to confirm the ferroelectric nature of our thin film as shown in Fig S3 (see Supplemental Material) [28]. 
In Figure 1(c), a drastic change in $\mathrm{P}_{\mathrm{r}}$ values from low $(\leq 20 \mathrm{kHz})$ to high $(\geq 40 \mathrm{kHz})$ frequencies was observed whereas there was little change in $\mathrm{P}_{\mathrm{S}}$ (the extrapolated value at $\mathrm{E}=0$ axis) as a function of frequency. The internal bias field created at each MOS structure will tend to stabilize polarization with opposite direction (see Figure 1(b)) due to their back-back connection. As such, at low frequency, our $\mathrm{Pt} / \mathrm{SnTiO}_{3} / \mathrm{Si} / \mathrm{SnTiO}_{3} / \mathrm{Pt}$ structure will suffer from backswitching phenomena resulting in very low net remnant polarization. However, at high frequency, the depletion will not play a significant role, so there will be negligible backswitching phenomena in the structure, resulting in high remnant polarization. We speculate that this is why we see a drastic change in $\mathrm{P}_{\mathrm{r}}$ in a stepwise fashion from $20 \mathrm{kHz}$ to $40 \mathrm{kHz}$, while we see small change in $\mathrm{P}_{\mathrm{s}}$ as a function of frequency in Figure 1(c).

Temperature dependent current (I) versus voltage (V) curves were measured from 83 to $600 \mathrm{~K}$ to understand the electrical conduction mechanism in $\mathrm{Pt} / \mathrm{SnTiO}_{3} / \mathrm{p}-\mathrm{Si} / \mathrm{SnTiO}_{3} / \mathrm{Pt}$ capacitors as shown in Fig. 2 (a). To reveal the dominant conduction mechanism of charge transport, the temperature dependent I-V curves were plotted on a logarithmic scale, as shown in Fig. 2 (b-c). As can be seen from Fig. 2 (c), the logarithmic I-V plot at room temperature can be fitted into three regimes, where the low and intermediate regimes are thought to arise from Ohmic conduction and Child's square law, respectively, and the highvoltage region corresponds to a steep increase in current [33]. Based on such I-V behavior, the conduction mechanism in our films is likely to be trap-assisted space charge limited current conduction [34]. However, it has often been observed in oxide thin films that a linear I-V relation at low applied voltages can also be explained by Simmons' modified Schottky emission mechanism. Unambiguous discrimination of these mechanisms will require thickness-dependent current measurements for further clarification [35]. At present, however, 
the temperature dependence of current can be used to determine the depth of the trapping levels. The slope of $\ln I$ versus $1 / T$, which is $\left(E_{c}-E_{t}\right) / k$, provides the activation energy value, as shown in Fig. 2 (d). We found that deep trap levels exist in the film with activation energy of $1.3 \pm 0.1 \mathrm{eV}$. This value is reasonable for oxygen-vacancy transport in perovskite oxides, including $\mathrm{SrTiO}_{3}$, where $1.1 \mathrm{eV}$ is the most common value [36, 37]. This value is found in two ways: From the ln I vs. (1000/T) graph in Fig. 2 (d) and, less precisely, as the kink and steep increase in current of the $\ln \mathrm{I}$ versus $\ln \mathrm{V}$ curve in Fig. 2 (c) near $\ln \mathrm{V}=0$ (i.e., $\mathrm{V}=1$ V).

It is important to rule out charge injection as a source of artifacts. In addition to noting that the polarization hysteresis loops are saturated, we provide in Fig. 3 data showing a dielectric peak at $\mathrm{T}_{\mathrm{C}}=450600 \mathrm{~K}$, depending upon probe frequency from ca. $500 \mathrm{~Hz}$ to 300 kHz. These data display the signature of a relaxor ferroelectric and satisfy a Vogel-Fulcher relationship with an extrapolated freezing temperature of ca. $452 \mathrm{~K}$ (Fig. 3b), suggesting local disordering of the Sn-ions. Inset of Fig. 3 (b) shows the relationship between dielectric constant and temperature near freezing temperature, which is described by modified CurieWeiss law [38]. A plausible hypothesis for the observed glassy behavior is the presence of some $\mathrm{Sn}^{4+}$ at the $\mathrm{Ti}^{4+}$ perovskite B-site. This could be tested via XPS measurement of the $\mathrm{Sn}^{4+} / \mathrm{Sn}^{2+}$ ratio. Since all these temperatures are well above ambient, these properties do not detract from the potential for commercial devices. Parenthetically, the Vogel-Fulcher behavior also helps rule out any artifacts as explanations of the hysteresis curves discussed above.

We have also observed the photovoltaic effect in $\mathrm{Pt} / \mathrm{SnTiO}_{3} / \mathrm{p}-\mathrm{Si} / \mathrm{SnTiO}_{3} / \mathrm{Pt}$ capacitors. In the dark, a very small current $\left(10^{-4} \mu \mathrm{A}\right)$ at zero bias voltage could be detected. Under white 
light illumination a distinct photovoltaic behavior was observed with short circuit current $\left(\mathrm{J}_{\mathrm{SC}}\right)=3 \mu \mathrm{A}$ and open circuit voltage $\left(\mathrm{V}_{\mathrm{OC}}\right)=0.13 \mathrm{~V}$, as shown in Fig. $4(\mathrm{a}) . \mathrm{V}_{\mathrm{OC}}$ and $\mathrm{J}_{\mathrm{SC}}$ were measured as a function of time over multiple on/off light cycles, showing good retention over time, with instability in $\mathrm{V}_{\mathrm{OC}}$ and $\mathrm{J}_{\mathrm{SC}}$ displaying a sudden increase and then return to initial values, as shown in Fig. 4 (b). To explain the unstable nature of $\mathrm{J}_{\mathrm{SC}}$ in multiple light on/off cycles, we measured the variation in $\mathrm{J}_{\mathrm{SC}}$ under continuous light illumination of 120 seconds, as shown in Fig. S4 (see Supplemental Material) [28]. We observed a continuous increase in photocurrent under continuous light illumination, which could be attributed to the presence of trap levels due to the structural defects, mainly oxygen vacancies $[39,40]$. Under the light illumination, photo-generated electrons are transferred to the conduction band and some of them occupy the deep trap levels. Photocurrent starts increasing slowly as the trap levels are filled gradually. In addition, we have also observed photosensitivity of our samples in the ultraviolet (UV) region. The photovoltaic behavior of $\mathrm{SnTiO}_{3}$ films under UV-light illumination has been shown in Fig. S5 (see Supplemental Material) $[28,41]$. There may be a small effect of the Si substrate on the photovoltaic effect but very limited as the photovoltaic effect from the $\mathrm{Si} / \mathrm{SnTiO}_{3}$ interfaces will be cancelled out due to the nature of back to back connection, and only the ferroelectric part will contribute to the photovoltaic effect as the ferroelectric polarization will be aligned in the direction from one Pt electrode to the other Pt electrode. To further confirm the contribution from $\mathrm{Si}$ substrate, we measured I-V curves under the illumination of a red laser pen $(\sim 650 \mathrm{~nm})$ and we observed a negligible contribution from $\mathrm{Si}$ in overall photovoltaic effect (see Supplemental Material, Fig. S6) [28]. 
Moreover, polarization fatigue, which is an important factor to determine the switchability of remnant polarization over the field cycles, was also measured with $10 \mu \mathrm{s}$ wide electric pulses at a frequency of $10 \mathrm{kHz}$. Figure 4 (c) shows the evolution of remnant polarization of $\mathrm{SnTiO}_{3}$ as a function of switching cycles. We confirmed nearly fatigueresistant characteristic up to $10^{6}$ cycles in our $\mathrm{SnTiO}_{3}$ capacitor, which is comparable to early studies of $\mathrm{Pt} / \mathrm{Pb}(\mathrm{Zr}, \mathrm{Ti}) \mathrm{O}_{3} / \mathrm{Pt}$ capacitors [42]. Interestingly, we have also observed photoferroelectric effect in $\mathrm{Pt} / \mathrm{SnTiO}_{3} / \mathrm{p}-\mathrm{Si} / \mathrm{SnTiO}_{3} / \mathrm{Pt}$ capacitor structures, where enhancement in ferroelectric properties was observed with an increase in $\mathrm{P}_{\mathrm{s}}$ and $\mathrm{P}_{\mathrm{r}}$ values under white light illumination. Such enhanced ferroelectric behavior shows the photo-ferroelectric nature of $\mathrm{SnTiO}_{3}$ thin films.

A combination of experimental and computational techniques was utilized to evaluate the optical band gap of $\mathrm{SnTiO}_{3}$. We measured the refractive indices of the film samples, $n$ and $k$, using spectroscopic ellipsometry technique and calculated absorption coefficient, $\alpha$. The (direct) band gap was determined to be $2.6 \mathrm{eV}$ by linearly extrapolating $(\alpha \mathrm{E})^{2}$ versus photon energy (E), as indicated by a gray line in Fig. 5 (a) [43].

In such oxides strain imposed by substrates could be an important factor to tailor the band gap. Electronic properties of perovskite $\mathrm{SnTiO}_{3}$, including band gap values, band structure and electronic density of states (EDOS), were investigated for varying epitaxial strain $\varepsilon$. Figure 5 (b)-(d) shows an electronic band dispersion diagram (left panel) and EDOS (right panel) of $\mathrm{SnTiO}_{3}$ obtained within the local density approximation (LDA) for (b) $\varepsilon=$ $-0.33 \%$, (c) $\varepsilon=0 \%$ and (d) $\varepsilon=+0.33 \%$, i.e., throughout the region where a transition from $\mathrm{P} 4 \mathrm{~mm}$ to $\mathrm{Cm}$ structure accompanied by a large band gap change was predicted by the previous investigation [12]. The band gap remains indirect (from $X$ to $\Gamma$ ) for all the 
considered strains, with value changing from $\sim 0.5$ (compression) to $1.0 \mathrm{eV}$ (tension), as previously reported [12]. We also included ion and $l$ quantum-number-resolved EDOS for the aforementioned three epitaxial strain states. Valence bands between 16 and $17 \mathrm{eV}$ are composed of Sn $s$ and $\mathrm{O} p$ states. Conduction bands between 19 and $21 \mathrm{eV}$ are largely composed of Ti $d$ states with a small mixture of $\mathrm{O} p$ states. It is well known that LDA exchange correlation functional underestimates the value of the band gap. We computed more accurate values of the perovskite $\mathrm{SnTiO}_{3}$ band gaps using the Heyd-Scuseria-Ernzerhof (HSE) screened hybrid functional (see Supplemental Material, Fig. S7) [28], obtaining values of $2.175 \mathrm{eV}$ for the $P 4 m m$ phase under compressive and zero strains, and $2.655 \mathrm{eV}$ for the $\mathrm{Cm}$ phase stabilized in epitaxial tension. The band gap value predicted by the HSE-based calculation for the monoclinic $\mathrm{Cm}$ phase is in a close agreement with the one determined by the experimental techniques, although at this point the nature of the band gap is not clear, since the experiment data point toward a direct band gap while the theory suggests an indirect band gap. The plot of strain dependent band gap based on EDOS suggests that we need to enhance tensile strain inside $\mathrm{SnTiO}_{3}$ film to further lower the optical band gap, which will enhance the photoferroelectric property of the film (see Supplemental Material, Fig. S7) [28].

\section{CONCLUSION}

In summary, we demonstrated room temperature relaxor ferroelectricity and photovoltaic effects in $\mathrm{SnTiO}_{3}$ thin films deposited directly on the p-type $\mathrm{Si}$ substrates. XRD data, polarization-electric field hysteresis loop and permittivity measurements, dependence of dielectric peaks at $\mathrm{T}_{\mathrm{C}}=450-600 \mathrm{~K}$ on probe frequency from ca. $500 \mathrm{~Hz}$ to $300 \mathrm{kHz}$, and a 
Vogel-Fulcher relationship with an extrapolated freezing temperature of ca. $452 \mathrm{~K}$, confirm the presence of relaxor ferroelectric perovskite $\mathrm{SnTiO}_{3}$ in our films. Furthermore, photovoltaic and photoferroelectricity were manifested in $\mathrm{Pt} / \mathrm{SnTiO}_{3} / \mathrm{Si} / \mathrm{SnTiO}_{3} / \mathrm{Pt}$ heterostructures, of which properties can be tuned through optical band gap engineering by strain according to the first-principles calculations. This study provides a path to develop green material for various ferroelectric and dielectric based emerging nanoelectronic devices and photoferroelectric applications.

\section{ACKNOWLEDGEMENTS}

R. A. acknowledges receiving graduate fellowships from NSF-IFN Grant \# 1002410. Work at Argonne (Y. S. and S. H., design of experiment, data analysis and contribution to manuscript writing) and ORNL (C.S. and H.N.L., optical spectroscopy; Y. S. ferroelectric characterizations) was supported by the U.S. Department of Energy (DOE), Office of Science, Office of Basic Energy Sciences (BES), Materials Sciences and Engineering

Division. S.C., K. P. and S. M. N. are grateful for financial support from the NSF (CDMR \#1309114), and C.G.T. acknowledges support from the NSF (CDMR \#1309114, CBET \#1067424 and EEC \#1062943). A part of the work was carried out at the University of Puerto Rico with financial support provided by the DoD-AFOSR Grant \# FA95501610295. J.F.S. acknowledges his visit expenses to UPR from NSF-IFN Grant \# 1002410. 


\section{REFERENCES}

1. Y. Konishi, M. Ohsawa, Y. Yonezawa, Y. Tanimura, T. Chikyow, H. Koinuma, A. Miyamoto, M. Kubo, and K. Sasata, Possible Ferroelectricity in $\mathrm{SnTiO}_{3}$ by FirstPrinciples Calculations, MRS Proceedings 748 (Materials Research Society, Pittsburgh, 2003), p.U3.13.1.

2. Y. Uratani, T. Shishidou, and T. Oguchi, First-Principles Predictions of Giant Electric Polarization, Jpn. J. Appl. Phys. 47, 7735 (2008).

3. S. Matar, I. Baraille, and M. Subramanian, First principles studies of $\mathrm{SnTiO}_{3}$ perovskite as a potential ferroelectric material, Chem. Phys. 355, 43 (2009).

4. T. Fix, S.-L. Sahonta, V. Garcia, J. L. M. Driscoll, M. G. Blamire, Structural and Dielectric Properties of $\mathrm{SnTiO}_{3}$, a Putative Ferroelectric, Cryst. Growth Des. 11, 1422-1426 (2011).

5. S. Chang, S. K. Selvaraj, Y.-Y. Choi, S. Hong, S. M. Nakhmanson, C. G. Takoudis, Atomic layer deposition of environmentally benign $\mathrm{SnTiO}_{x}$ as a potential ferroelectric material, J. Vac. Sci. Technol. A 34, 01 A119 (2016).

6. I. P. Batra, P. Wurfel, and B. D. Silverman, New type of first-order phase transition in ferroelectric thin films, Phys. Rev. Lett. 30, 384-387 (1973).

7. M. Dawber, P. Chandra, P. B. Littlewood, J. F. Scott, Depolarization corrections to the coercive field in thin-film ferroelectrics, J. Phys.: Condens. Matter 15, L393-398 (2003).

8. L. Pintilie, M. Alexe, Ferroelectric-like hysteresis loop in nonferroelectric systems, Appl. Phys. Lett. 87, 112903 (2005).

9. J. F. Scott, Ferroelectric Memories (Springer, Heidelberg, 2000). 
10. P. Sirajudheen and K. M. Muhammed Ismayil, Synthesis Characterization and Photocatalytic Activity of SnTiO3 Prepared by Co-Precipitation Peroxide Method, Chem. Sci. J. 6, 100111 (2015).

11. K. S. Beenakumari, Visible Light driven Tin Titanate Photo-Catalyst Prepared by CoPrecipitation Method, Int. J. Mater. Sci. Innovation 1, 174-181 (2013).

12. W. D. Parker, J. M. Rondinelli, S. M. Nakhmanson, First-principles study of misfit strain-stabilized ferroelectric $\mathrm{SnTiO}_{3}$, Phys. Rev. B 84, 245126 (2011).

13. M. F. M. Taib, M. K. Yaakob, O. H. Hassan, M. Z. A. Yahya, Structural, Electronic, and Lattice Dynamics of $\mathrm{PbTiO}_{3}, \mathrm{SnTiO}_{3}$, and $\mathrm{SnZrO}_{3}:$ A Comparative FirstPrinciples Study, Integr. Ferroelectr. 142, 119-127, (2013).

14. V. M. Fridkin, Photoferroelectrics (Springer, 1979).

15. J. Kreisel, M. Alexe, P. A. Thomas, A photoferroelectric material is more than the sum of its parts, Nat. Mater. 11, 260 (2012).

16. S. Liu, F. Zheng, I. Grinberg, A. M. Rappe, Photoferroelectric and Photopiezoelectric Properties of Organometal Halide Perovskites, J. Phys. Chem. Lett. 7, 1460 - 1465 (2016).

17. R. Agarwal, Y. Sharma, and R. S. Katiyar, Switchable photovoltaic and polarization modulated rectification in Si-integrated $\mathrm{Pt} /\left(\mathrm{Bi}_{0.9} \mathrm{Sm}_{0.1}\right)\left(\mathrm{Fe}_{0.97} \mathrm{Hf}_{0.03}\right) \mathrm{O}_{3} / \mathrm{LaNiO}_{3}$ heterostructures, Appl. Phys. Lett. 107, 162904 (2015).

18. P. Wang, J. Zhao, L. Wei, Q. Zhu, S. Xie, J. Liu, X. Meng, J. Li, Photo-induced ferroelectric switching in perovskite $\mathrm{CH}_{3} \mathrm{NH}_{3} \mathrm{PbI}_{3}$ films, Nanoscale 9, 3806 (2017).

19. P. Hohenberg, W. Kohn, Inhomogeneous Electron Gas, Phys. Rev. 136, B864 (1964). 
20. W. Kohn, L. J. Sham, Self-Consistent Equations Including Exchange and Correlation Effects, Phys. Rev. 140, A1133 (1965).

21. G. Kresse, J. Furthmüller, Efficient iterative schemes for ab initio total-energy calculations using a plane-wave basis set, Phys. Rev. B 54, 11169 (1996).

22. G. Kresse, J. Furthmüller, Efficiency of ab-initio total energy calculations for metals and semiconductors using a plane-wave basis set, Comput. Mater. Sci. 6, 15 (1996).

23. J. P. Perdew, A. Zunger, Self-interaction correction to density-functional approximations for many-electron systems, Phys. Rev. B 23, 5048 (1981).

24. P. E. Blöchl, Projector augmented-wave method, Phys. Rev. B 50, 17953 (1994).

25. G. Kresse, D. Joubert, From ultrasoft pseudopotentials to the projector augmentedwave method, Phys. Rev. B 59, 1758 (1999).

26. H. J. Monkhorst, J. D. Pack, Special points for Brillouin-zone integrations, Phys. Rev. B 13, 5188 (1976).

27. J. Heyd, G. E. Scuseria, M. Ernzerhof, Hybrid functionals based on a screened Coulomb potential, J. Chem. Phys. 118, 8207 (2003).

28. See Supplemental Material at [URL] for x-ray diffraction analysis, capacitancevoltage measurements, temperature dependent polarization hysteresis loop measurements, photocurrent transient measurement, photovoltaic characterization, and band gap tuning via tensile strain in $\mathrm{SnTiO}_{3}$ thin films.

29. M.F.M. Taib, M.K. Yaakob, A. Chandra, A.K. Arof, M.Z.A. Yahya, Effect of Pressure on Structural, Electronic and Elastic Properties of Cubic (Pm3m) $\mathrm{SnTiO}_{3}$ Using First Principle Calculation, Adv. Mat. Res. 501, 342-346 (2012).

30. L. Pintilie, C. Dragoi, R. Radu, A. Costinoaia, V. Stancu, and I. Pintilie, Temperature induced change in the hysteretic behavior of the capacitance-voltage characteristics of $\mathrm{Pt}-\mathrm{ZnO} \mathrm{O}-\mathrm{Pb}\left(\mathrm{Zr}_{0.2} \mathrm{Ti}_{0.8}\right) \mathrm{O}_{3}-\mathrm{PtPt}-\mathrm{ZnO}-\mathrm{Pb}\left(\mathrm{Zr}_{0.2} \mathrm{Ti}_{0.8}\right) \mathrm{O}_{3}-\mathrm{Pt}$ heterostructures, Appl. 
Phys. Lett. 96, 012903 (2010).

31. L. Pintilie, M. Lisca, and M. Alexe, Polarization reversal and capacitance-voltage characteristic of epitaxial $\mathrm{Pb}(\mathrm{Zr}, \mathrm{Ti}) \mathrm{O}_{3} \mathrm{~Pb}(\mathrm{Zr}, \mathrm{Ti}) \mathrm{O}_{3}$ layers, Appl. Phys. Lett. 86, 192902 (2005).

32. W. Choi, S. Kim, Y. W. Jin, S. Y. Lee, and T. D. Sands, Capacitance-voltage modeling of metal-ferroelectric-semiconductor capacitors based on epitaxial oxide heterostructures, Appl. Phys. Lett. 98, 102901 (2011).

33. Y. Sharma, P. Misra, R. S. Katiyar, Unipolar resistive switching behavior of amorphous $\mathrm{YCrO}_{3}$ films for nonvolatile memory applications, J. Appl. Phys. 116, 084505 (2014).

34. M. A. Lampert, P. Mark, Current Injection in Solids (Academic, New York, 1970).

35. J. F. Scott, There's no place like Ohm: conduction in oxide thin films, J. Phys.: Condens. Matter 26, 142202 (2014).

36. J. F. Scott, Ferroelectric Memories (Springer, Heidelberg, 2000).

37. R. Meyer, R. Liedke, R. Waser, Oxygen vacancy migration and time-dependent leakage current behavior of $\mathrm{Ba}_{0.33} \mathrm{Sr}_{0.7} \mathrm{TiO}_{3}$ thin films, Appl. Phys. Lett. 86, 112904 (2005).

38. K. Uchino and S. Nomura, Critical Exponents of the Dielectric Constants in DiffusedPhase-Transition Crystals, Ferroelectrics Lett., 44, 55-61 (1982).

39. R. Moubah, O. Rousseau, D. Colson, A. Artemenko, M. Maglione, M. Viret, Photoelectric Effects in Single Domain $\mathrm{BiFeO}_{3}$ Crystals, Adv. Funct. Mater. 22, 4814-4818 (2012).

40. D. Mahgerefteh, J. Feinberg, Explanation of the apparent sublinear photoconductivity of photorefractive barium titanate, Phys. Rev. Lett. 64, 2195 (1990). 
41. M. Alexe, D. Hesse, Tip-enhanced photovoltaic effects in bismuth ferrite, Nat. Commun. 2, 256 (2011).

42. E. L. Colla, S. Hong, D. V. Taylor, A. K. Tagantsev, N. Setter, K. No, Direct observation of region by region suppression of switchable polarization fatigue in $\mathrm{Pb}(\mathrm{Zr}, \mathrm{Ti}) \mathrm{O}_{3}$ thin film capacitors with Pt electrodes, Appl. Phys. Lett. 72, 2763-2765 (1998).

43. S. Hong, E. Kim, D.-W. Kim, T.-H. Sung, and K. No, On measurement of optical band gap of chromium oxide films containing both amorphous and crystalline phases, J. Non-Cryst. Solids 221, 245 - 254 (1997). 


\section{FIGURE CAPTIONS}

Figure 1 (a) Polarization-electric field (P-E) hysteresis loop and switching current at different applied voltages between 1 and $10 \mathrm{~V}$ measured at room temperature. Inset shows the direction of hysteresis loop as a function of time. (b) Schematic diagram of $\mathrm{Pt} / \mathrm{SnTiO}_{3} / \mathrm{p}$ $\mathrm{Si} / \mathrm{SnTiO}_{3} / \mathrm{Pt}$ capacitor structure used for electrical measurements. (c) Frequency dependence of $\mathrm{P}$ E hysteresis loops.

Figure 2 (a) $\mathrm{I}-\mathrm{V}$ characteristics of $\mathrm{Pt} / \mathrm{SnTiO}_{3} / \mathrm{p}-\mathrm{Si} / \mathrm{SnTiO}_{3} / \mathrm{Pt}$ capacitors at various temperatures. (b) $\operatorname{lnI}-\ln \mathrm{V}$ plots at various temperatures. (c) $\ln \mathrm{I}-\ln \mathrm{V}$ plot at $300 \mathrm{~K}$ exhibits trap assisted space charge limited current (SCLC) behavior of current transportation. (d) Arrhenius plot for capacitor structure.

Figure 3 (a) Temperature dependence of the dielectric constant. (b) Extrapolated freezing temperature was observed to be $452 \mathrm{~K}$. Inset shows the linear fitting of experimental data obtained from modified Curie-Weiss law. We note that for any relaxor, as the probe frequency is lowered, the temperature corresponding to dielectric constant peaks must decrease and the peak magnitude must increase; in any viscous material the susceptibility (both electrical and mechanical) must increase as frequency decreases.

Figure 4 (a) $\mathrm{I}-\mathrm{V}$ characteristics of $\mathrm{Pt} / \mathrm{SnTiO}_{3} / \mathrm{p}-\mathrm{Si} / \mathrm{SnTiO}_{3} / \mathrm{Pt}$ capacitor in dark and under white light illumination. Inset shows the portion of the I-V curves around zero field, revealing $\mathrm{J}_{\mathrm{SC}}$ and $\mathrm{V}_{\mathrm{OC}}$ to be $3 \mu \mathrm{A}$ and $0.13 \mathrm{~V}$, respectively. (b) Time dependence of $\mathrm{J}_{\mathrm{SC}}$ and $\mathrm{V}_{\mathrm{OC}}$ was measured under multiple on/off light cycles. (c) Evolution of remnant polarization 
with the number of bipolar cycles in dark and light. Inset shows the P-E hysteresis loops after $10^{6}$ switching cycles.

Figure 5 (a) $(\alpha \mathrm{E})^{2}$ versus photon energy plot gives the direct band gap of $2.6 \mathrm{eV}$ for $\mathrm{SnTiO}_{3}$ thin films grown on p-Si substrate. A gray line is an extrapolation line to estimate the band gap. (b-d) Electronic bands dispersion (left panel) and ion and $l$ quantum-number-resolved electronic density of states (EDOS) (right panel) calculated for $\mathrm{SnTiO}_{3}$ for the biaxial misfit strain $\varepsilon$ of (b) $-0.33 \%$, (c) $0 \%$ and (d) $0.33 \%$ within the local density approximation (LDA). The location of the bands is referenced with respect to the low-lying $\mathrm{O} 2 s$ state, which is assumed to be undisturbed by any structural distortions. 


\section{FIGURES}

(a)

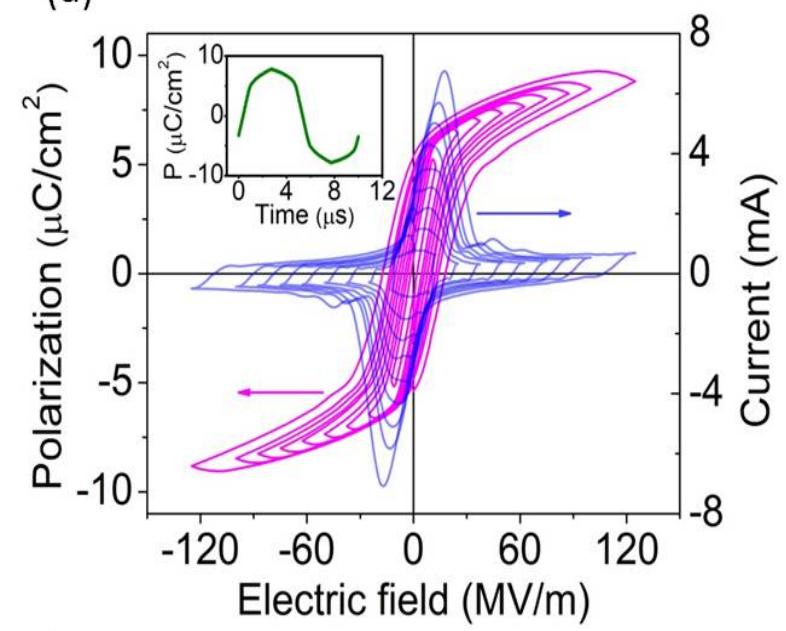

(b)

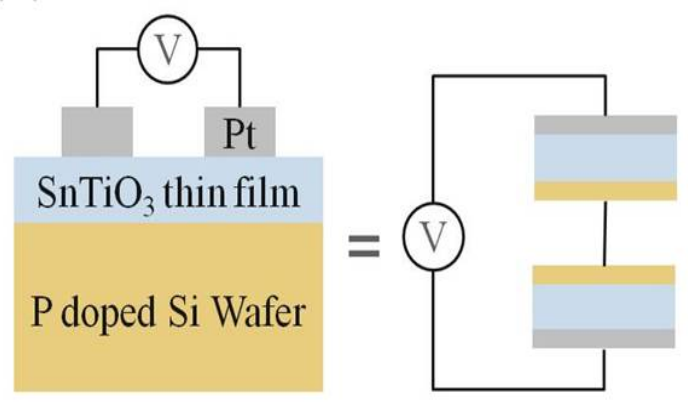

(c)

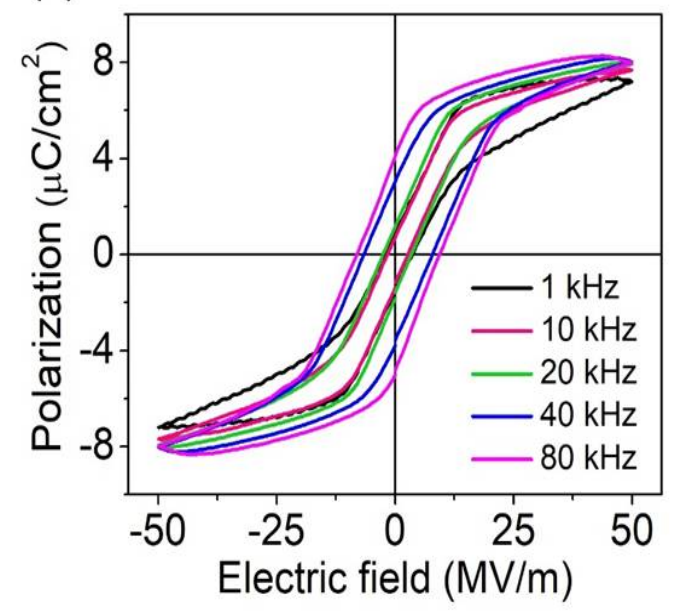

Figure 1 
(a)

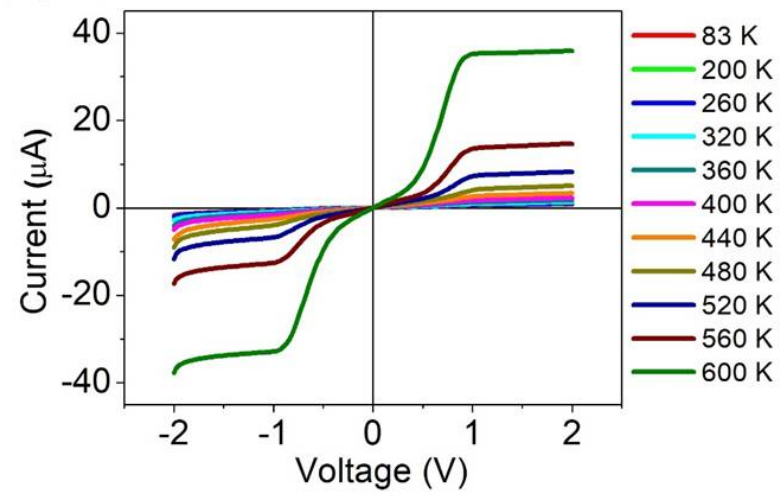

(c)

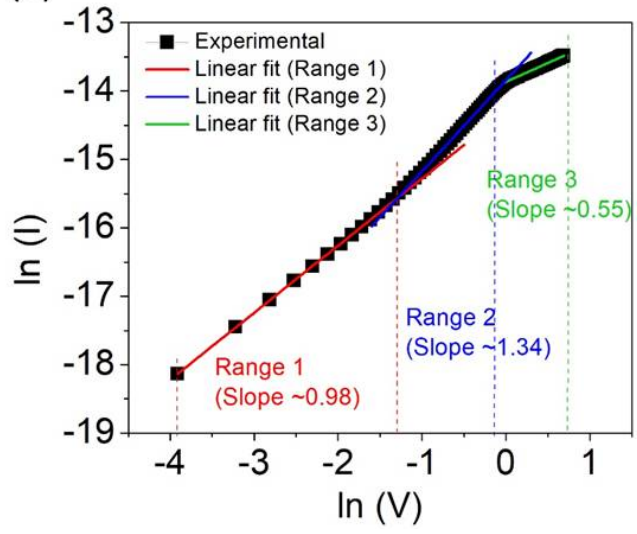

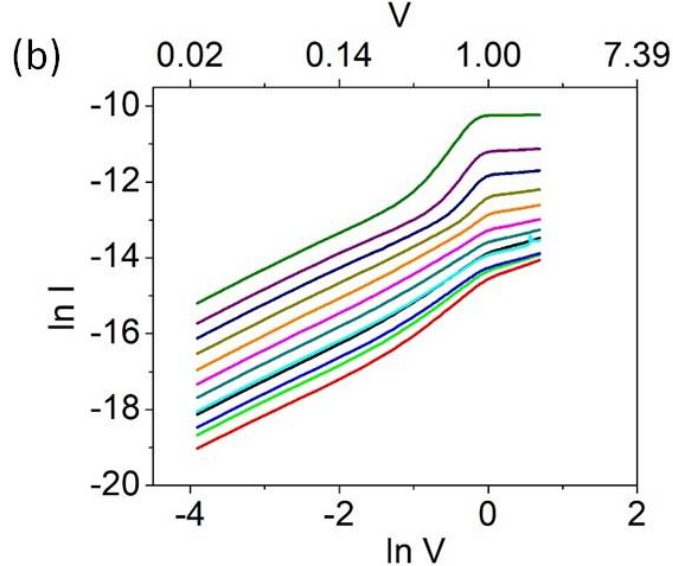

$\begin{array}{llll}\text { (d) } \quad 500 \quad 417 & \text { T (K) } \\ & 557 & 313\end{array}$

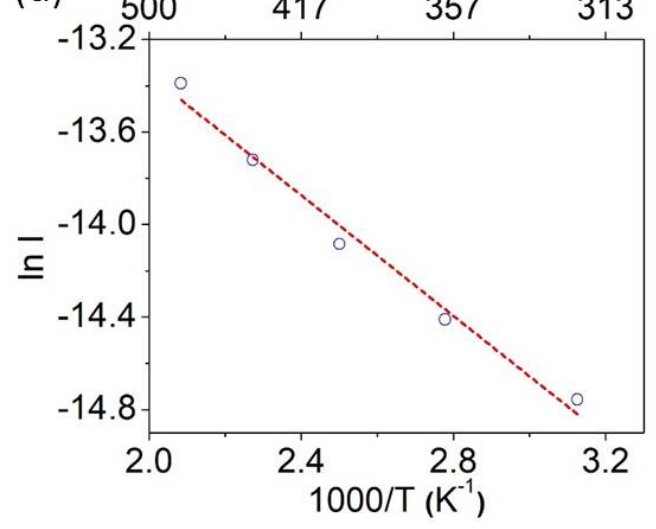

Figure 2 

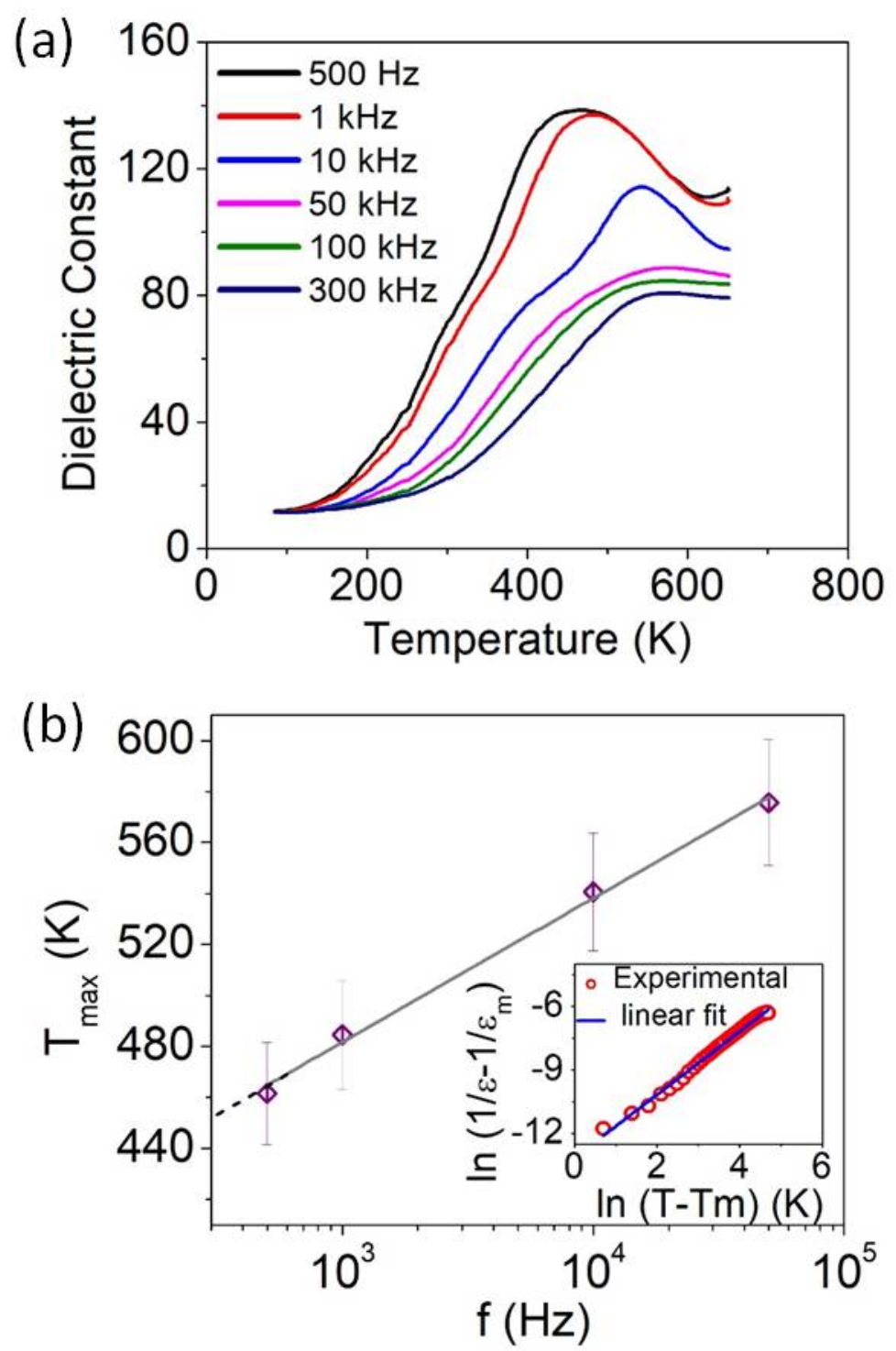

Figure 3 
(a)

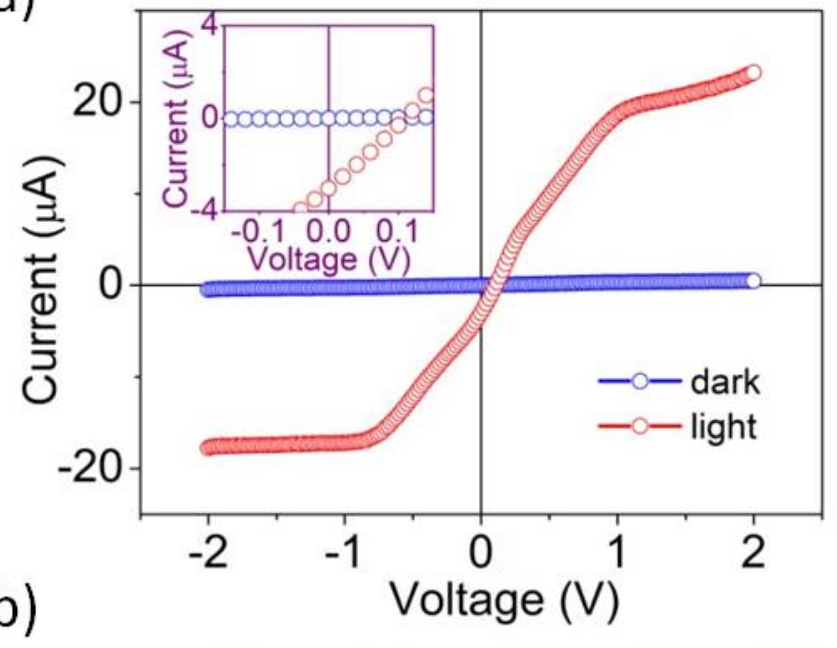

(b)
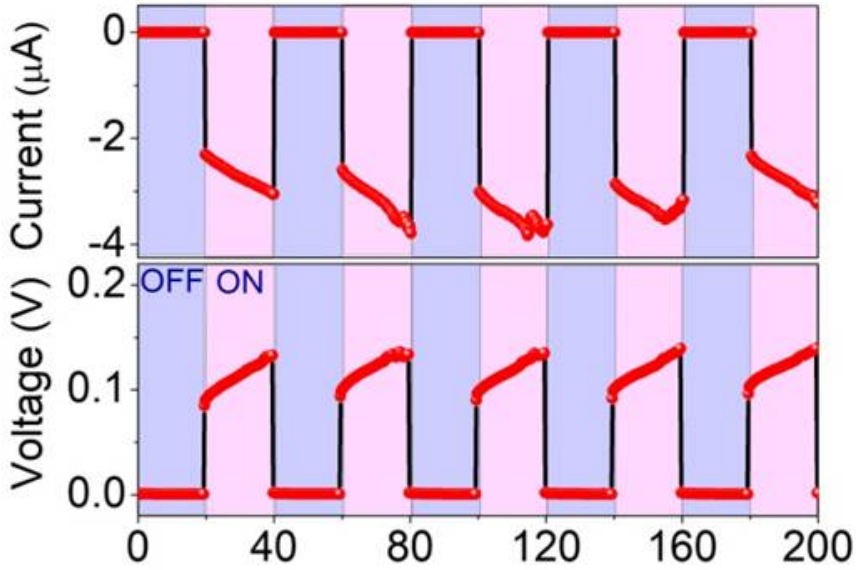

(c)

Time (s)

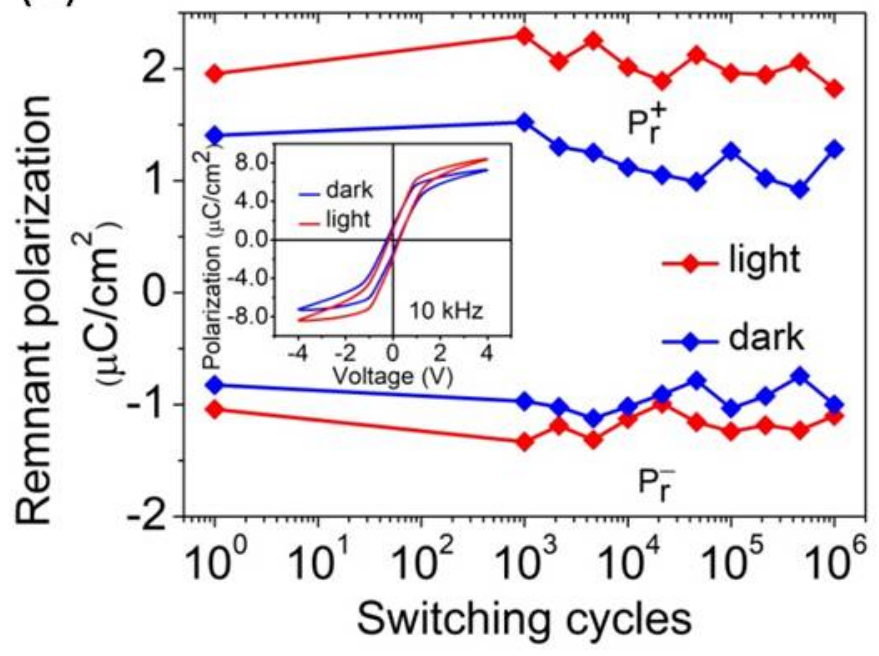

Figure 4 

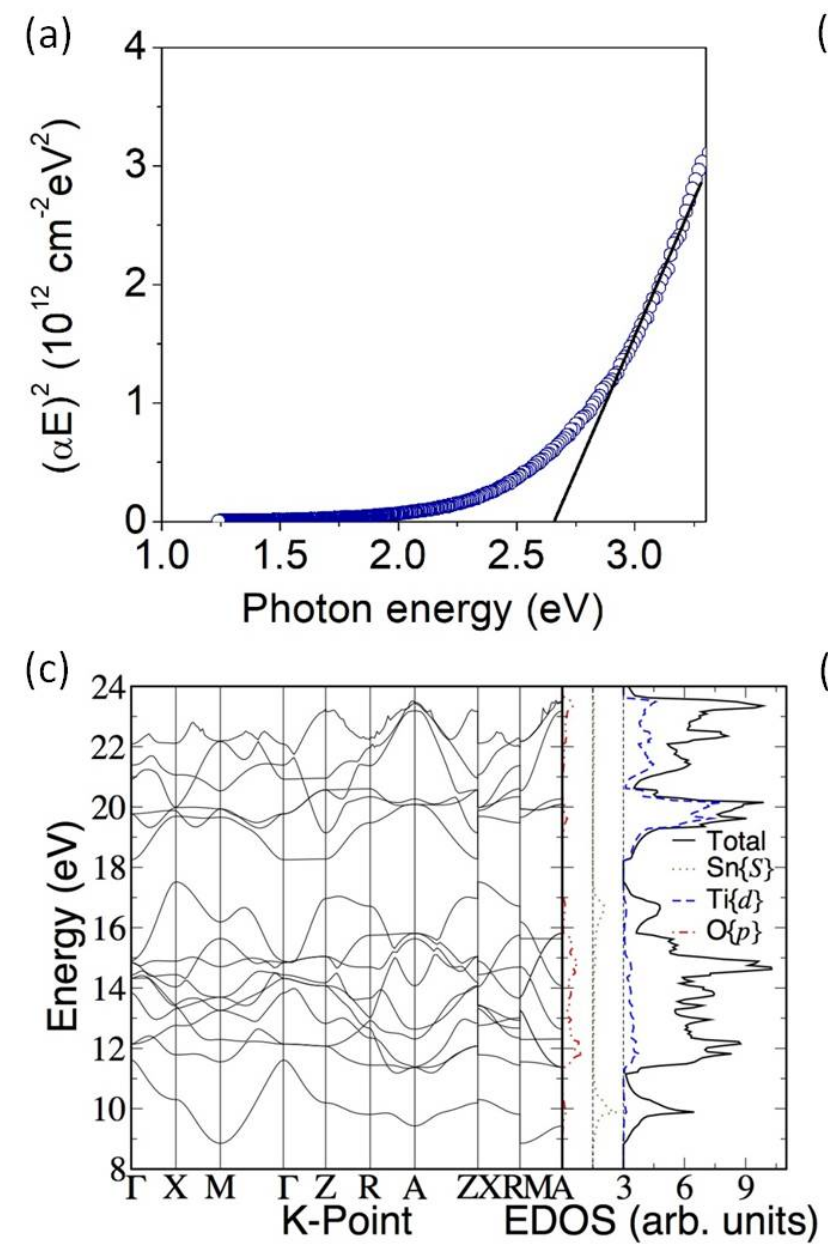
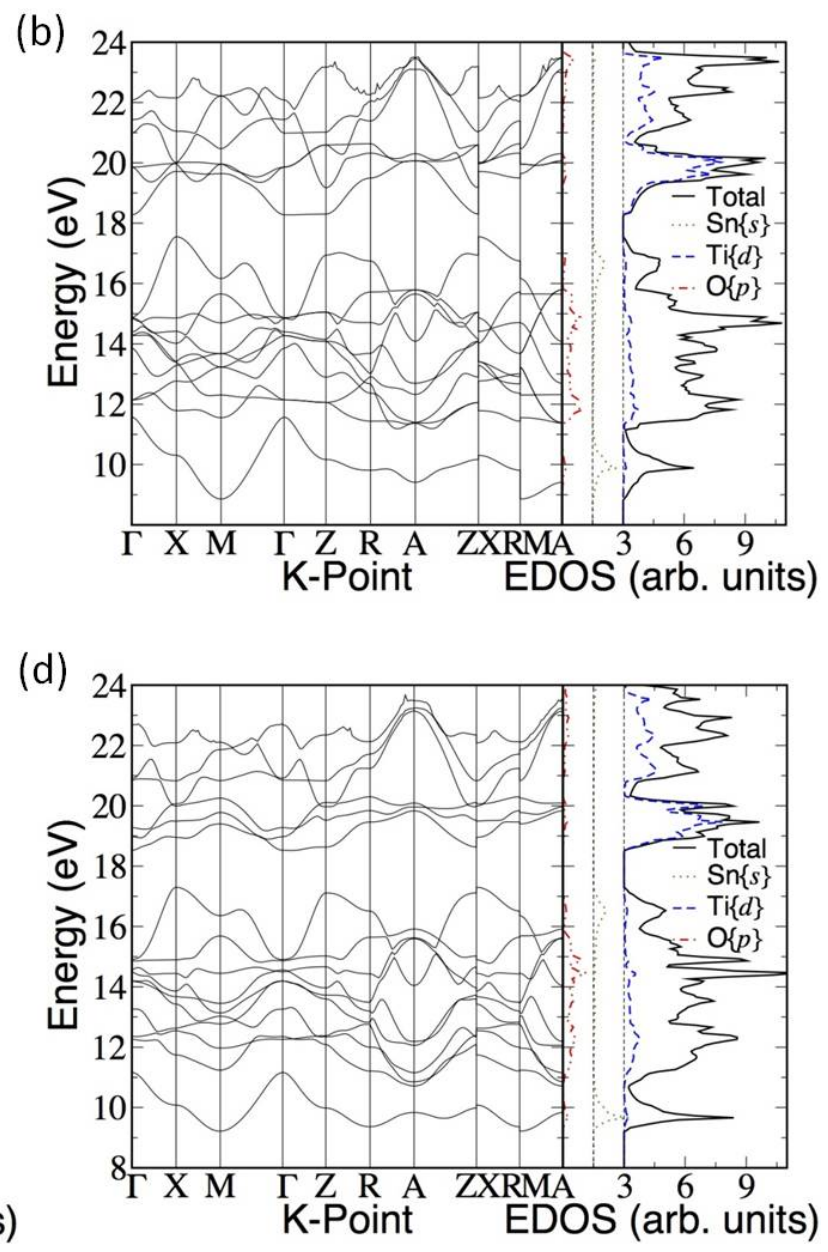

Figure 5 